\title{
A Novel Multi-Working Electrode Potentiostat for Electrochemical Detection of Metabolites
}

\author{
Daniela De Venuto*, Michele Daniel Torre \\ Dipartimento di Elettrotecnica ed Elettronica \\ Politecnico di Bari \\ Bari, Italy \\ *d.devenuto@poliba.it
}

\author{
Cristina Boero, Sandro Carrara, Giovanni De Micheli \\ Laboratory of Integrated Systems \\ Swiss Federal Institute of Technology (EPFL) \\ Lausanne, Switzerland
}

\begin{abstract}
A novel single-chip and multiplexed read-out circuit for multi-electrode electrochemical sensors, in standard $0.18 \mu \mathrm{m}$ UMC CMOS technology, is presented. The circuit is a part of a fully-integrated biochip (in design) for the detection of multiple metabolites. The proposed topology is based on the potentiostat approach, and it is devoted to detect currents within the range of $250 \mathrm{pA}-650 \mathrm{nA}$ for an electrode active area of $0.25 \mathrm{~mm}^{2}$. The need of multi-metabolites monitoring asks for a system with multi-working electrodes. In the proposed configuration, switches select one working electrode at each clock phase, while the others are short-circuited to the reference one, in order to nullify the injected current inside the counter. Low noise and low energy topology $(50 \mu \mathrm{W}$ at $1.5 \mathrm{~V}$ of voltage supply) is employed for the control amplifier. The linearity of the proposed read-out circuit allows accuracy better than $0.1 \%$.
\end{abstract}

\section{INTRODUCTION}

Monitoring metabolic compounds, such as glucose, lactate and glutamate, is of extreme importance in medical diagnostics for many purposes [1-6]. In the last 50 years, a wide effort has been directed to the development of amperometric enzyme biosensors to detect glucose in human blood, mainly driven by the demand coming from diabetic pathology. On the other hand, the research can also be extended to the detection of other compounds, like endogenous metabolites and exogenous drugs in the blood or in the interstitial fluid. One of the challenges in this field is the detection of multiple compounds in the same time, in order to either deeply understand biological mechanisms, or to develop devices for personalized therapies. The integration of multiple sensors to detect many compounds in the same time, and the integration with the required electronics, can pave the way to smart systems used in clinical diagnostics.

Recently, a new demand for these kinds of devices is also emerging from the field of cell analysis, in order to monitor specific analytes involved in cell differentiation and growth. Glucose is the most interesting metabolite, since it is a necessary nutrient for almost the totality of cell cultures. Lactate monitoring is also interesting, since it is related to hypoxia and cell stress [1]. Glutamate is an another relevant analyte involved in cell development because it is the major neurotransmitter in the brain. Thus, it is particularly useful to monitor neural cells [2]. The contemporary monitoring of such metabolites can be a further instrument for the investigation of cell mechanisms, which are still not clear. It should be of special importance to monitor differentiation processes, especially in the case of embryonic or mesenchimal stem cells.

Until now, the application of biosensors to cell monitoring by metabolic compounds released by cells in culture media has not been largely explored yet. On the other hands, new systems for real-time monitoring are required to succeed in the field of system biology. Nayak et al. [3] employed two commercially available devices for blood glucose selfmonitoring to evaluate off-line glucose concentration in culture medium. The work consists in a recalibration of the two chosen systems in order to fit within the range of glucose in cell culture medium. Rodrigues et al. [5] developed a miniaturized cell-based biochip dedicated to analysis of cells during their growth. The Rodrigues' system is an electrochemical sensors integrated with inlet and outlet microchannels made of PDMS (polydimethylsiloxane). So, a cell chamber is realized to perform real time and continuous monitoring of glucose and oxygen during cell culturing. Potentiostat was not integrated into the chip, but wires connected the electrodes to an external one. Recently, Pemberton et al. [6] developed an amperometric biosensor to monitor a liver cell line. Also in this case, detection was performed with off-line measurements.

All the aforementioned examples show the monitoring of few process variables, like glucose and $\mathrm{O}_{2}$. In the present work, we want to extend the concept of potentiostat in order to design and realize a fully integrated biochip for the detection of multiple metabolites by means of on-line measurements. In this paper, the multiplexed read-out circuit for multi-working electrode potentiostat is presented. Current range and circuit sensitivity has been evaluated from current-glucose concentration curves measured with electrodes having an active area of the electrode equal to $2 \mathrm{~mm}^{2}$. For these measurements, electrodes were nanostructurated with Multi- 
Walled Carbon Nanotubes (MWCNT) and glucose oxidase. Studies have demonstrated that CNTs promote the electrontransfer between the enzyme redox active site and the transducing electrode [6]. Those values of current were scaled for an active area of $0.25 \mathrm{~mm}^{2}$, in order to miniaturize the whole system. The circuit is designed and its performance is verified through simulations.

\section{THE MULTI-ELECTRODES SENSOR}

Fig. 1 shows a possible layout for the multi-electrodes sensor that allows the sensing of multiple metabolites. The figure shows five working electrodes, one reference, and one large counter. In the following, the detailed description of the sensor and of the calibration set-up is given.

\section{Chemicals}

Gold screen-printed electrodes (SPE - model DRPC223AT) and multi-walled carbon nanotubes were purchased from Dropsens (Spain). The electrodes are made of a gold working electrode, which presents an active area equal to 2 $\mathrm{mm}^{2}$, a counter electrode, also made of gold, and a reference electrode, which is made of silver. The total area of the cell is $22 \mathrm{~mm}^{2}$. Multi-walled carbon nanotubes (diameter $10 \mathrm{~nm}$, length $1-2 \mu \mathrm{m}$ ) were purchased in powder (90\% purity), and subsequently diluted in chloroform to the concentration of 1 $\mathrm{mg} / \mathrm{ml}$ [8]. Samples were then sonicated in order to obtain a homogeneous solution. Glucose oxidase from Aspergillus Niger (GOD, EC 1.1.3.4, 129.9 units/mg solid) and D-(+)glucose were purchased from Sigma-Aldrich (Switzerland) in lyophilized powder. The protein was dissolved in Phosphate Buffer Saline (PBS) solution 0.01 M at pH 7.4, while glucose was dissolved in Milli-Q.

\section{Preparation of electrodes}

Nanostructured SPEs were prepared by using MWCNT and the probe enzyme. To prepare the MWCNT modified SPE, $6.5 \mu \mathrm{l}$ of the MWCNT-chloroform solution was deposited by drop-casting $(0.5 \mu \mathrm{l}$ each time $)$ onto the working electrode and it is allowed to dry. Then, $3.2 \mu \mathrm{l}$ of glucose oxidase $(15 \mathrm{mg} / \mathrm{ml})$ were dropped onto the working electrode and stored overnight at $+4^{\circ} \mathrm{C}$ in order to allow the adsorption of the proteins onto the electrode surface. Then, the drop was rinsed out with Milli-Q and the electrode was conditioned for 10 minutes at constant potential $(+550 \mathrm{mV})$ before the first use. The electrode was covered with PBS and stored at $+4^{\circ} \mathrm{C}$, when not used.

\section{Apparatus}

The electrochemical response of electrodes is investigated by chronoamperometry under aerobic conditions. Electrochemical measurements were acquired by using Versastat 3 potentiostat (Princeton Applied Technologies). The electrode was dipped into the PBS solution with a volume of $25 \mathrm{ml}$ under stirring conditions. A volume of $25 \mu \mathrm{l}$ per step of the target molecule was successively added into the solution with a time-step of 2 minutes.

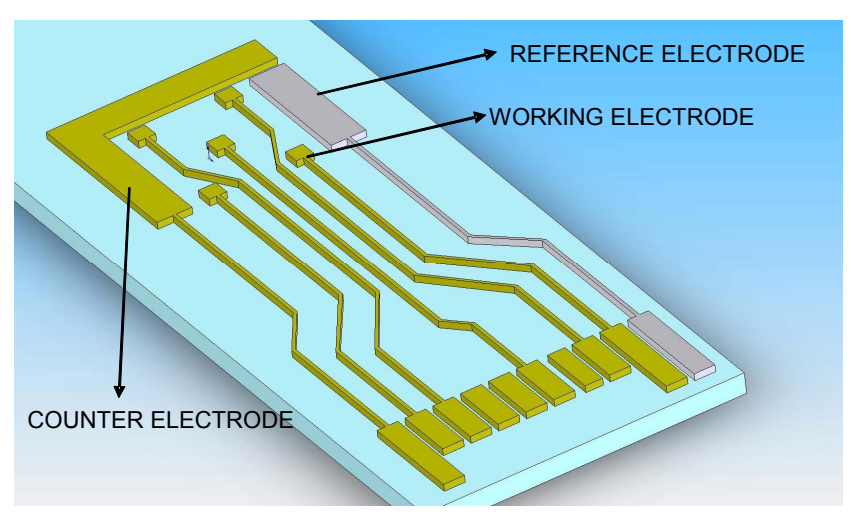

Figure 1 Layout of the multiple metabolites sensor.

\section{Sensor calibration}

Improvements of nanostructured electrodes by using MWCNT were previously demonstrated in the case of glucose and lactate for working electrodes with area of about 13 $\mathrm{mm}^{2}$ [9]. For the present study, calibration curve is worked out from working electrodes with an active area of $2 \mathrm{~mm}^{2}$, in order to investigate the signal magnitude from the electrochemical cell when scaling down working electrode dimensions. Calibration curve is investigated by chronoamperometry within a concentration range of from 0.5 to $4 \mathrm{mM}$. The concentration range is chosen to be suitable for measurements in cell cultures. Glucose is dissolved in Milli-Q and added into the PBS solution every $120 \mathrm{~s}$, to allow the system to reach the steady-state. The response time of the system after each addition is around $30 \mathrm{~s}$ (data not show). The calibration curve in the case of glucose detection shows a sensitivity of $9.3 \mu \mathrm{A} \mathrm{mM} \mathrm{mm}^{-1} \mathrm{~cm}^{-2}$, as shown in Fig. 2 .

Electrodes size-scaling was considered in order to investigate $\mathrm{v}$ further developments on integration in a single chip. For that aim, the considered working electrodes of the chip model presents an active area around $0.25 \mathrm{~mm}^{2}$ (see Fig.1).

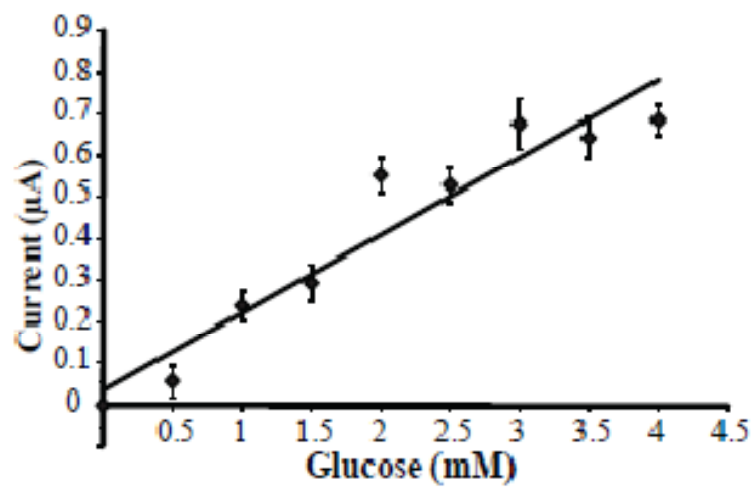

Figure 2 Calibration curve for glucose detection in the concentration range from 0.5 to $4 \mathrm{mM}$ 


\section{BIOCHIP ARCHITECTURE}

The proposed chip is based on an extension of the usually considered three-electrode electrochemical cell. It contains the usual counter and reference electrodes, and different working electrodes (e.g., $\mathrm{WE}_{1}, \mathrm{WE}_{2}$, and $\mathrm{WE}_{3}$ ), required for detection of different metabolites. The reference electrode (RE) keeps constant the potential of the solution while the current is collected from the counter electrode (CE). An analog multiplexer selects the working electrodes at each clock cycle. The architecture for the three working electrodes topology is shown in Fig. 3. Of course, this design is also suitable to be easily expanded with more working electrodes.

The potentiostat configuration has been used for stabilizing the voltage at the each working electrode (WEi) and nullify the current in the reference electrode, accordingly with well established literature on three-electrode cell configurations [10]. The potential on the RE must be stable for reducing fluctuations in current measurements [11]. In the chosen topology (Fig. 3), based on the potential control loop introduced by M. Ahamadi [10], the WE has been kept to a fixed potential, thank to the series-shunt feedback loop and to the high loop gain. A current-to-frequency converter has been used to digitalize the information and improve the quality of the current reading. A detailed description of the architecture blocks will be given in the sequel.

\section{A. Analog Multiplexer}

Referring to Fig. 3, $\mathrm{CK}_{1}, \mathrm{CK}_{2}, \mathrm{CK}_{3}$ are the CMOS switches related to $\mathrm{WE}_{1}, \mathrm{WE}_{2}$, and $\mathrm{WE}_{3}$, respectively. They are activated on the positive edge of the clock. Instead, $\overline{C K}_{1}$, $\overline{C K}_{2}$, and $\overline{C K}_{3}$ are the switches activated on the negative clock edge. The $\overline{C K}_{1}, \overline{C K}_{2}$, and $\overline{C K}_{3}$ allow the current trapped inside the parasitic capacitance of the switches to be discharged through the counter. If the WE1 has to be considered, CK1 has to be on, while $\mathrm{CK}_{2}$ and $\mathrm{CK}_{3}$ are off. Consequently, a potential is applied on the WE1. On the same time, $\overline{C K}_{1}$ will be off, while $\overline{C K}_{2}$ and $\overline{C K}_{3}$ will be switched-on. Since the input current of the operational amplifier is negligible, due to the high gain, also the current in $\overline{C K}_{2}$ and $\overline{C K}_{3}$ will be almost zero. An automatic control of the switches can be made using synchronized clock signal. Fig. 3 on the top right, shows the clock signals for the switches $\mathrm{CK}_{1}, \mathrm{CK}_{2}$ and $\mathrm{CK}_{3}$.

\section{B. Model of the electrochemical cell}

The current range for the chip design has been chosen on the basis of the calibration curve of glucose detection (Fig. 2) acquired with a sensor having an active area equal to $2 \mathrm{~mm}^{2}$. In chronoamperometry, we can consider the Cottrell equation [4]:

$$
i=\frac{n F A D^{1 / 2} C}{(\pi t)^{1 / 2}}
$$

where $\boldsymbol{i}$ is the current (A), $\mathbf{n}$ is the number of electrons, $\mathbf{F}$ is Faraday's constant, $\mathbf{A}$ is the electrode area $\left(\mathrm{cm}^{2}\right), \mathbf{D}$ is the diffusion coefficient of the analyte $\left(\mathrm{cm}^{2} / \mathrm{sec}\right), \mathbf{C}$ concentration

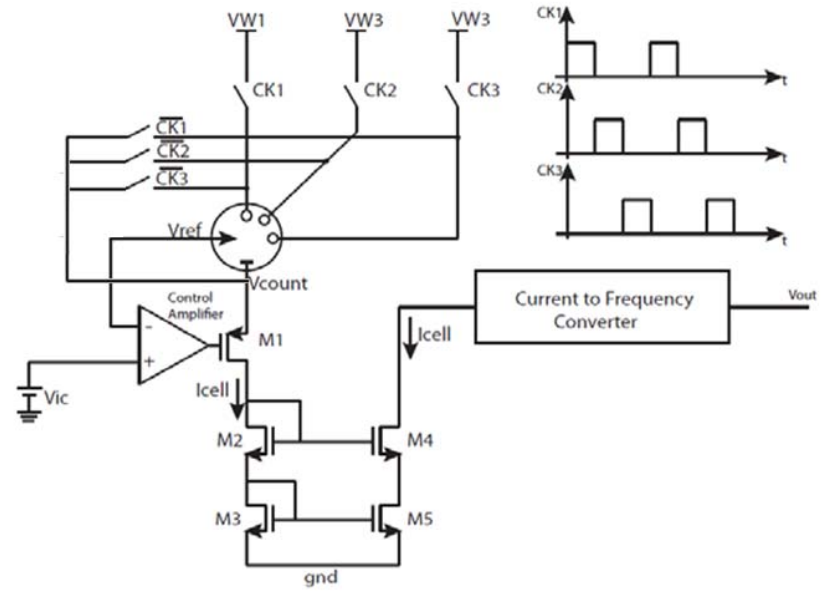

Figure 3 Multi-electrodes (three in the present configuration) potentiostat, with multiplexer and clock timing

of the analyte $\left(\mathrm{mol} / \mathrm{cm}^{3}\right)$, and $\mathrm{t}$ is time of the diffusion. From the calibration curve in Fig. 2, we can estimate a sensitivity for the $2 \mathrm{~mm}^{2}$ sensor of $9.32 \mu \mathrm{A} /\left(\mathrm{mM} \cdot \mathrm{cm}^{2}\right)$. As shown in (1), there is a linear relationship between current flowing in the counter electrode and the working electrode area. Then, a current range from $6 \mathrm{nA}$ up to $550 \mathrm{nA}$ has been evaluated for electrodes with area equal to $0.25 \mathrm{~mm}^{2}$, taking into account concentration ranges measured in cell culture [9].

For multi-working measurements, the electrochemical cell has been modeled according to the scheme in fig. 4 [12]. $\mathrm{R}_{\mathrm{S} 1}$ and $\mathrm{R}_{\mathrm{S} 2}$ represent the solution impedances, which are typically small (about $190 \mathrm{ohm}$ ) and $\mathrm{R}_{\mathrm{Ref}}$ is the reference electrode resistance. $\mathrm{R}_{\text {Count }}, \mathrm{R}_{\mathrm{Work} 1}, \mathrm{R}_{\mathrm{Work} 2}, \mathrm{R}_{\text {Work3 }}$ represent electrode resistances, and $\mathrm{C}_{\text {Count }}, \mathrm{C}_{\text {Work } 1}, \mathrm{C}_{\text {Work2 }}, \mathrm{C}_{\text {Work3 }}$ are the double-layer capacitances associated with the $\mathrm{CE}$ and the variously WEs, respectively. The model capacitances value have been derived by the one used in [13]. In order to adapt our configuration to the three electrodes topology, the equivalent circuits of the cell has been considered accordingly with reference [14].

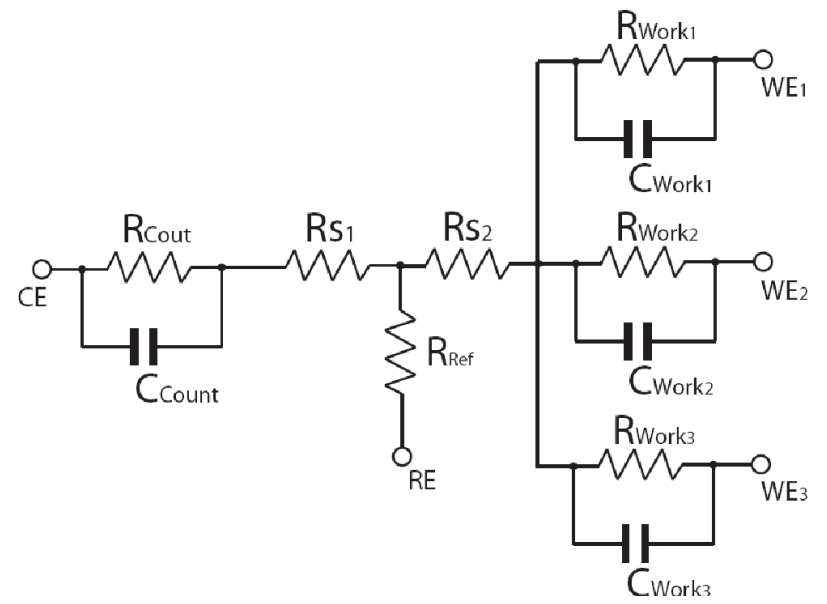

Figure 4 The three WE's electrochemical cell model 
The double-layer capacitances are given by:

$$
C=k \cdot A
$$

where $\mathrm{C}$ is the capacitance of the electrode, $\mathrm{A}$ is the area of the electrode, and $\mathrm{k}$ is the ratio between permittivity of the electrode-solution interface and the thickness. The used capacitance value is of $\mathrm{C}_{\mathrm{Work}}=1.2 \mu \mathrm{F}$, and has been scaled for an electrode of $0.25 \mathrm{~mm}^{2}$ active area. Following [14], the value coming from the $\mathrm{Ohm}$ low is considered for $\mathrm{R}_{\text {Work, }}$ :

$$
R_{W o r k}=\frac{V_{c e l l}}{I_{f}}
$$

where $V_{\text {cell }}$ is the electrode potential, and $I_{\mathrm{f}}$ is the current collected by the electrode. For glucose measurements, the Vcell is usually set at $550 \mathrm{mV}$ and the current range of $\mathrm{I}_{\mathrm{f}}$, derived by the current scaling on the calibration curve in Fig. 2 , is considered from $6 \mathrm{nA}$ to $550 \mathrm{nA}$. In this way, an $\mathrm{R}_{\text {Work }}$ ranging from $1.2 \mathrm{M} \Omega$ to $110 \mathrm{M} \Omega$, has been obtained from equation (3). For $\mathrm{R}_{\text {Count }}$ and $\mathrm{C}_{\text {Count }}$ the values reported in literature [10] have been used.

\section{Control Amplifier}

A potentiostat has been used for stabilizing the voltage on the RE. The potential on the RE must be stable for reducing fluctuations in current measurements [11]. In literature is possible to find different kinds of potentiostat for electrochemical cells presenting two [14] o three electrodes [11]. However, better performances are achieved with the three-electrode configuration [11]. The proposed circuit performs chronoamperometry acquisitions on oxidative currents. In the chosen topology, the WE has been fixed to a potential making it insensitive to noise and interference pickup [11]. Fig. 3 also shows the potentiostat block diagram. The operational amplifier realizes a voltage stabilizer. Changes in the current flowing into the counter electrode induce potential variations on the reference electrode. Then, the potential on the reference electrode is kept constant thank to the negative feedback (series-shunt) of the op-amp and to the high loop gain (see Fig. 3). Variations on the RE are cancelled by changing the potential on the MOS M1 gate and driving back to positive input voltage value (Vic) the Vref. The MOS M1 has a low threshold voltage to keep the amplifier in the linearity region, and modulates the very little current in $\mathrm{CE}$ toward equality in the inputs potentials of the control amplifier.

A current mirror is used to reduce the input-referred noise voltage. We can consider the voltage on the drain as the output of the considered block, and $\mathrm{R}_{\mathrm{D}}$ as the current mirror output resistance. If we consider only the noise on $R_{D}$, namely the $\left|V_{n}, R_{D}\right|$, then the input-referred noise $\left|V_{n, \text { in }}\right|$ is given by:

$$
\left.\left|V_{n, i n}\right|=\frac{\left|V_{n, R D}\right|}{A_{1} R_{D}} \mid \frac{1}{g_{m}}+\left(1+A_{1}\right) R_{S}\right]
$$

where $A_{1}$ is the op-amp gain, $g_{m}$ is the MOS transconductance, and $\mathrm{R}_{\mathrm{S}}$ is the resistance at the source of M1 (i.e. the cell resistance). Equation (4) shows that $\left|V_{n, i n}\right| \rightarrow\left|V_{n}, R_{D}\right| R_{S} / R_{D}$ if $A 1 \rightarrow \infty$ ( $A_{1}$ is extremely high). In our case, the op-amp open-loop gain is $72 \mathrm{~dB}$ and $\mathrm{R}_{\mathrm{D}}$ is given by $2 / g_{m}$, the gain of the reference branch of the current mirror (see Fig.3). This does not make the system completely immune to noise and it is the reason why multiplexing improves the circuit performance.

\section{Current-to-frequency converter}

The current-to-frequency converter has been realized using the circuit shown in Fig. 5. The converter reads the current coming from the current mirror and converts it into a periodic voltage signal, which has the frequency directly proportional to the current value. The operating principle is based on the charging and discharging of capacitor $\mathrm{Cp}$, generating a sawtooth signal in input of the two op-amp, which work as comparators. One comparator is performing the coarse comparison having as reference $600 \mathrm{mV}$ while the second is performing the fine one respect to a threshold at $60 \mathrm{mV}$. The output latch performs the regenerative action in asynchronous mode [16].

The time for charging or discharging the capacitor $\Delta \mathrm{T}$ is given by:

$$
\Delta T=C_{p} \frac{\Delta V}{I_{c o n v}}
$$

where $\Delta \mathrm{V}$ is the voltage swing and $\mathrm{I}_{\text {conv }}$.is the cell current to be detected by the I-f conversion.

To understand the device behavior, let us consider initially the capacitor Cp charged and the MOS M29 off. Then, the capacitor discharges through the mirror resistance. When the voltage value at the M29 drain reaches the reference value at the negative input of the high gain comparator, (Vdown, in Fig. 5), then the transistor M29 switches on and charges again

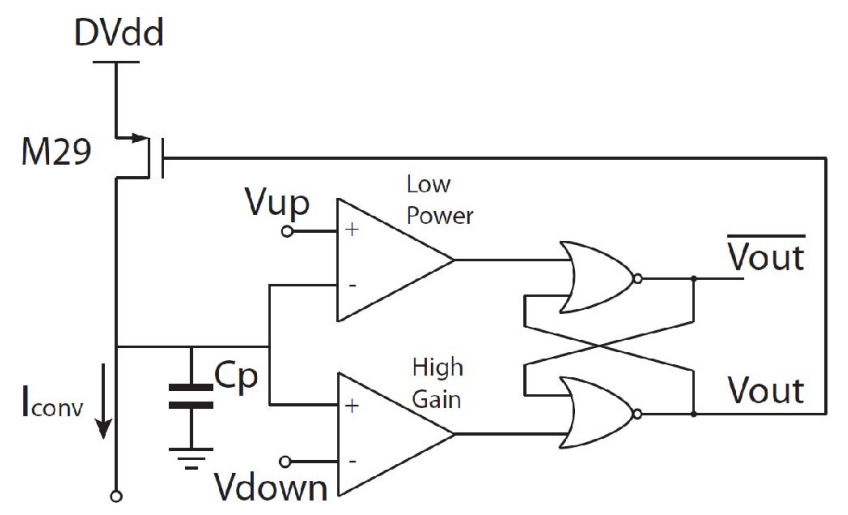

Figure 5 Current to frequency converter scheme 
the capacitor $\mathrm{Cp}$. A second and low power comparator has been inserted with a S-R flip flop for keeping the MOS M29 closed until the capacitor is fully charged. So, the discharge time will be inversely proportional to the mirror current, accordingly to equation (5). A high value of the capacitor $\mathrm{Cp}$ enables a low-pass filtering of the noise but produces a longer discharging time.

\section{Simulation Results}

In this session, the Cadence tool simulation results on the multielectrodes system are presented. Fig. 6 shows the I-to-f converter behavior for a single measurement, i.e. when a single working electrode is enabled, by considering for $\mathrm{Cp}$ a value equal to $1 \mathrm{pF}$. Fig. 6a shows the voltage on the capacitor $\mathrm{Cp}$ at the comparators input while Fig. $6 \mathrm{~b}$ shows the converter output. The analysis has been performed keeping at a fixed potential of $704 \mathrm{mV}$ the working electrode, the Reference at $700 \mathrm{mV}$ and considering Rcell equal to $2 \mathrm{M} \Omega$ for a cell current of Icell equal to $1.936 \mathrm{nA}$. When the value of $\mathrm{Vcp}$ reaches $600 \mathrm{~m}$, then the Vout goes to $1.5 \mathrm{~V}$. This generates a current spike in M29 and, thus, in Vout.

The Fig. 7 shows the calibration curve of this current-tofrequency conversion. In the figure, is the results of simulations are compared with a regression line in order to show the quality of the converter. A linearity of $0.1 \%$ of was estimated for the performance of the whole system. This linearity fully satisfies the accuracy requested for the considered applications.

Fig. 8 shows the simulation results on multiplexing the three working electrodes included in the potentiostat-based system shown in Fig. 3. The figure shows from the top, the current in M1 MOS (see Fig. 3), the counter electrode voltage, the reference voltage biasing the working electrode at the clock edge, and the WE1, WE2, WE3 voltages when selected.

The simulations shows that even when the WE3 is selected for the current measure, then small spikes appear on the other working and reference electrodes due to clocking.
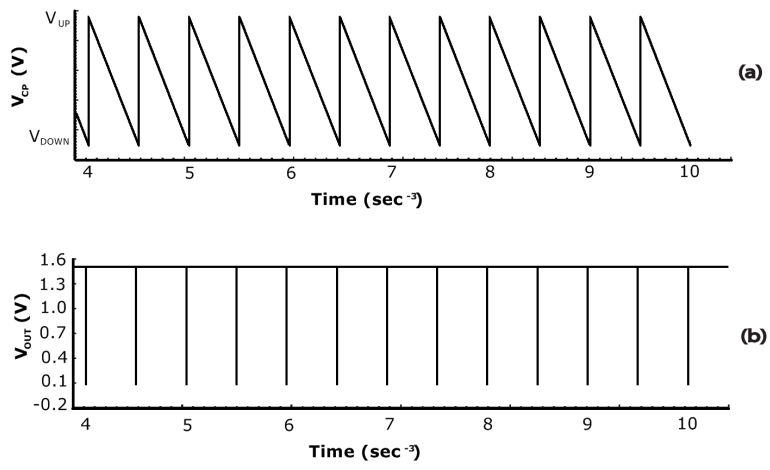

Figure 6 I-f Conversion for the single electrode:

(a) the voltage at the input of the comparator;

(b) the comparator output.

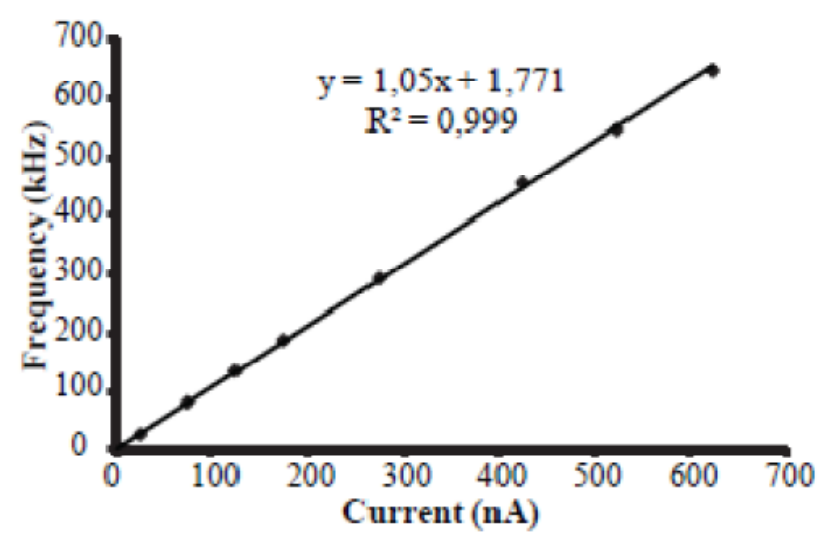

Figure 7 The calibration curve showing also the regression error respect the theoretical curve.

Being the signal analysis quite slow (range of $\mathrm{Hz}$ ) the spikes do not introduce artifacts in the current estimation and they could be even reduced by cancelling the charge injection.

The currents values measured for a single electrode system and for the multielectrodes here proposed are proposed by comparison in table 1 . The sensor cell has been biased with a Vcell of $0.55 \mathrm{~V}$ and the capacitance Ccell is here considered equal to $1.2 \mathrm{uF}$, while the resistance has been changed as shown in table 1 in order to emulate the current changing in the electrochemical cell. The measured currents values are very similar in comparing the working performances of the single electrode system with those of the multi-electrodes one. The measured values are coherent with those estimated by theoretical computations (last column in table 1) with a maximum relative error of $0.7 \%$.

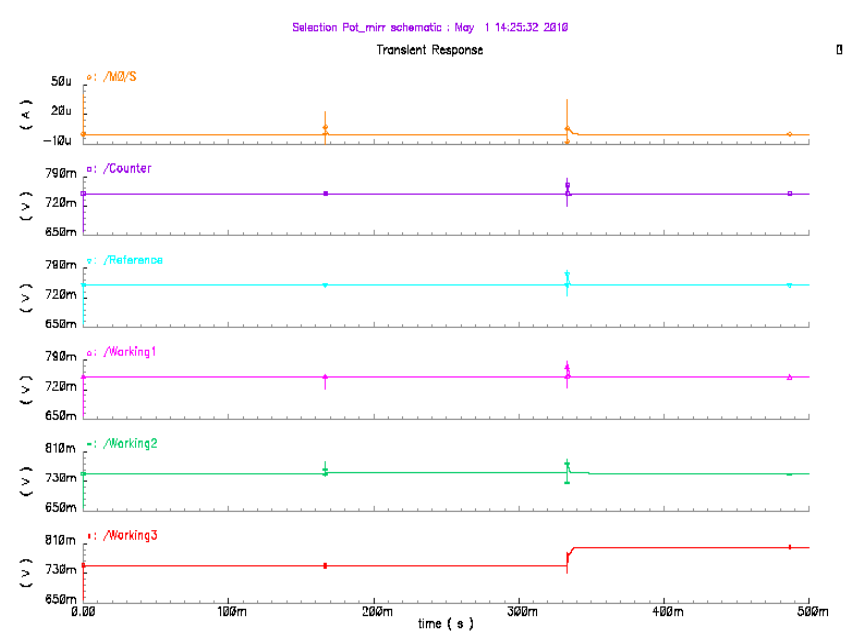

Figure 8 The three electrodes potentiostat-based system simulations 
Table 1 Electrodes Currents

\begin{tabular}{|l|l|l|l|}
\hline $\begin{array}{l}\text { Rcell } \\
{[\mathrm{M} \Omega]}\end{array}$ & $\begin{array}{l}\text { Single } \\
\text { electrode } \\
{[\mathrm{A}]}\end{array}$ & $\begin{array}{l}\text { Multielectrode } \\
\text { current } \\
{[\mathrm{A}]}\end{array}$ & $\begin{array}{l}\text { Theoretical } \\
\text { Electrode } \\
\text { current }[\mathrm{A}]\end{array}$ \\
\hline 2 & $274.9 \mathrm{n}$ & $273 n$ & $275 n$ \\
\hline 5 & $109.9 n$ & $109.7 \mathrm{n}$ & $110 \mathrm{n}$ \\
\hline 10 & $54.9 n$ & $54.9 n$ & $55 n$ \\
\hline 50 & $11 n$ & $11 n$ & $11 n$ \\
\hline 100 & $5.499 n$ & $5.499 n$ & $5.5 n$ \\
\hline 200 & $2.749 n$ & $2.75 n$ & $2.75 n$ \\
\hline 300 & $1.83 n$ & $1.83 n$ & $1.83 n$ \\
\hline 400 & $1.37 n$ & $1.37 n$ & $1.37 n$ \\
\hline 500 & $1.1 n$ & $1.1 n$ & $1.1 n$ \\
\hline
\end{tabular}

\section{CONCLUSION}

A novel single-chip and multiplexed read-out circuit for multi-electrode electrochemical sensors, in standard $0.18 \mu \mathrm{m}$ UMC CMOS technology, is presented in this paper. The circuit is a component to achieve the final goal of a fully integrated biochip for detection of multiple metabolites in invitro cell cultures or in in-vivo tissues for applications in personalized therapy. The proposed topology is based on the potentiostat approach, and it is focused on detecting currents within the range from $250 \mathrm{pA}$ up to $650 \mathrm{nA}$, in case of electrodes active area of $0.25 \mathrm{~mm}^{2}$. The multi-metabolites monitoring asks for a system with multiple working electrodes. In the proposed configuration, switches select one working electrode for each clock phase, while the others are short-circuited to the counter in order to nullify the injected current . Low noise topology $(50 \mu \mathrm{W}$ at $1.5 \mathrm{~V}$ of voltage supply) is proposed for the control amplifier. The linearity of the read-out circuit allows us to achieve an accuracy better than $0.1 \%$ that fully satisfy the application requests.

\section{ACKNOWLEDGMENT}

This research has been supported by Swiss National Science Foundation by means of the Sinergia project CRSII2_127547 / 1 and by a Sino-Swiss cooperation project $n$. IZLCZ2 123967.

\section{REFERENCES}

[1] N. Nikolaus, and B. Strehilitz, "Amperometric lactate biosensors and their application in (sport) medicine, for life quality and wellbeing," Microchim.Acta, vol. 160, pp. 15-55, 2008.

[2] S. Qin, M. van der Zeyden, W. H. Oldenziel, T. Cremers, and B. Westeink, "Microsensors for in vivo measurement of glutamate in brain tissue," Sensors, vol. 8, pp. 6860-6884, 2008.

[3] R. C. Nayak, and I. M. Herman, "Measurement of glucose consumption by hybridoma cells growing in hollow fiber cartridge bioreactors: use of blood glucose self-monitoring devices," J. Immunol. Methods., vol. 205, pp. 109-114, 1997.

[4] A.J. Bart, and L.R. Faulkner, Electrochemical Methods, Foundamentals and Applications, John Wiley and sons inc. NY, Second Edition, 2001, pag. 163

[5] N. P. Rodrigues, Y. Sakai, and T. Fujii, "Cell-based microfluidic biochip for the electrochemical real-time monitoring of glucose and oxygen," Sensor Actuat. B-Chem., vol. 132, pp. 608-613, 2008.

[6] R. M. Pemberton, J. Xu, R. Pittson, N. Biddle, G. A. Drago, S. K. Jackson, J. P. Hart, "Application of screen-printed microband biosensors to end-point measurements of glucose and cell numbers in HepG2 cell culture," Anal. Biochem., vol. 385, pp. 334-341, 2009.

[7] C. Cai, and J. Chen, "Direct electron transfer of glucose oxidase promoted by carbon nanotubes," Anal. Biochem., vol. 332, pp. 75-83, 2004.

[8] S. Carrara, V. V. Shumyantseva, A. I. Archakov, and B. Samorí, "Screen-printed electrodes based on carbon nanotubes and cytochrome p450scc for highly sensitive cholesterol biosensors," Biosens. Bioelectron., vol. 24, pp. 148-150, 2008.

[9] C. Boero, S. Carrara, G. Del Vecchio, G. D. Albini, L. Calzà, G. De Micheli, "Carbon nanotubes-based electrochemical sensing for cell culture monitoring," IEEE International Conference on Complex Medical Engineering 2010, Gold Coast, Australia.

[10] M. Ahmadi and G. Jullien, "Current-mirror-based potentiostats for three-electrode amperometric electrochemical sensors," IEEE Transactions on Circuits and Systems Part I: Regular Papers, vol. 56, pp. 1339-1348, 2009

[11] M. M. Ahmadi and G. A. Jullien,, K. Iniewski, Ed., "Circuits for amperometric electrochemical sensors," in VLSI Circuit Design for Biomedical Applications. Norwood, MA: Artech House, 2008.

[12] Shumyantseva, V.V., et al., Direct electron transfer between cytochrome P450scc and gold nanoparticles on screen-printed rhodium-graphite electrodes. In Biosensors and Bioelectronics, 2005. 21(1): p. 217-222.

[13] H. Narula and J. Harris, "A time-based VLSI potentiostat for ion current measurements," IEEE Sensors Journal, vol. 6, pp. 239-247, 2006

[14] S. M. R. Hasan: "Stability analysis and novel compensation of a cmos current-feedback potentiostat circuit for electrochemical sensors". 7(5):814\{824, 2007

[15] Steven M. Martin at all.: "A Fully Differential Potentiostat" in IEEE Sensor Jounal vol. 9, 2, pp.135-142 Feb. 2009

[16] D. De Venuto, E. Stikvoort, Y. Ponomarev, D. Castro: "Ultra LowPower 12-bit SAR ADC for RFID Applications", Proc. Of IEEE Design and Automation and Test in Europe (DATE2010) Dresden March 2010. 